\section{Inquérito epidemiológico sobre hipertensão arterial em Volta Redonda - RJ}

* Escola Nacional de Saúde Pública - FIOCRUZ Rio de Janeiro
At the end of the year 1979 a cross-seccional study on arterial hypertension was carried out in the town of Volta Redonda. Households, as epidemiologic units, were randomically sorted. 650 adults from 20 to 74 years old were examined at home. The current paper describes in detail the methodology used for collecting the information and some of the results are presented.

The mean systolic and diastolic pressures were respectively $122.4 \pm 0.9$ and $70.6 \pm 0.7 \mathrm{mmHg}$. The prevalence of hypertension according to the WHO criteria (systolic $\geqslant 160$ and/or diastolic $\geqslant 95 \mathrm{mmHg}$ ) was $10.1 \pm$ 1.3 per cent, considering the effect of the household cluster sampling design.

In Volta Redonda higher prevalence of arterial hypertension were found in young and middle-age groups. This probably explains the high mortality ratio by diseases of the cardio-vascular system found in the above age groups.

\title{
INTRODUÇÃO
}

Volta Redonda é um município do estado do Rio de Janeiro, Brasil, situado às margens do rio Paraíba do Sul, onde está instalada a Usina Presidente Vargas da Companhia Siderúrgica Nacional, inaugurada em 1946, da qual depende praticamente toda a economia do município. O local foi escolhido para sede da usina devido a sua localização entre os dois maiores centros urbanos do país, São Paulo e Rio de Janeiro. A população do município em 1980, segundo dados preliminares do censo, era de 183.917 habitantes, dos quais 180.420 moravam na cidade $(98 \%)$ e 3.515 na área rural (2\%). ${ }^{8}$ Em 1970, segundo dados censitários, cerca de $50 \%$ das pessoas tinham menos de 20 anos de idade ${ }^{7}$. Em 1980 , a razão sexual era de, praticamente, 1 homem para 1 mulher (92.307 e 91.610 , respectivamente) ${ }^{8}$. Em relação à ocupação somente os dados de 1970 são disponíveis, indicando estes que $2 / 3$ dos homens, com 10 anos ou mais, estavam trabalhando na indústria, ao passo que o mesmo era observado para $1 / 10$ das mulheres na mesma faixa etá- 
ria ${ }^{7}$. Volta Redonda é, pois, uma cidade tipicamente indus-

trial, porém de marcada concentração na indústria siderúrgica.

Através de um levantamento dos dados existentes sobre causas de óbitos, nos anos do final da década de 60 e início de $70^{19}$, verifica-se que, consideradas as diferenças de estruturas etárias, a mortalidade por causas cardiovasculares, e especificamente por doenças hipertensivas, é bastante elevada em Volta Redonda, sendo quase o dobro da mortalidade por estas causas no estado inteiro, e, até mesmo sensivelmente maior do que a do Rio de Janeiro, o segundo pólo urbano do país (tabela I). Observa-se, também, que, no mesmo período, um quarto dos óbitos em Volta Redonda eram atestados pelos médicos como devidos a causas cardiovasculares, o que pode ser considerado como uma proporção elevada dadas a distribuição etária da população, com predominância marcada de pessoas com menos de 40 anos, e a imprecisão de causas registradas em $1 / 5$ dos atestados de óbito (tabela II).

\section{TABELA I}

Mortalidade bruta e padronizada(1), por 100000 habitantes, por causas cardiovasculares e por todas as causas, e mortalidade proporcional por sintomas e estados mórbidos mal definidos no estado do Rio de Janeiro, no pertodo de 1968-1972.

\begin{tabular}{|c|c|c|c|c|c|c|c|c|}
\hline \multirow[b]{2}{*}{ Causas de Óbitos } & \multicolumn{2}{|c|}{ Volta Redonda } & \multicolumn{2}{|c|}{ Niterói } & \multicolumn{2}{|c|}{$\begin{array}{l}\text { Município } \\
\text { Rio de Janeiro }\end{array}$} & \multicolumn{2}{|c|}{ Estado do Rio } \\
\hline & Bruta & Padron. & Bruta & Padron. & Bruta & Padron. & Bruta & Padron. \\
\hline $\begin{array}{l}\text { Febre Reumática Ativa } \\
\text { Doença Reum. Crôn. do Coração } \\
\text { Doenças Hipertensivas } \\
\text { Doenças Isquêmicas Coração } \\
\text { Outras Formas de Doença Cor. } \\
\text { Doença Cerebrovascular }\end{array}$ & $\begin{array}{r}0.32 \\
3.99 \\
34.00 \\
38.63 \\
46.13 \\
76.30\end{array}$ & $\begin{array}{r}0.39 \\
5.26 \\
53.51 \\
61.20 \\
75.56 \\
123.26\end{array}$ & $\begin{array}{r}0.80 \\
7.90 \\
38.98 \\
100.05 \\
89.56 \\
113.43\end{array}$ & $\begin{array}{r}0.83 \\
7.21 \\
32.22 \\
83.12 \\
76.40 \\
95.66\end{array}$ & $\begin{array}{r}0.98 \\
6.71 \\
37.92 \\
116.06 \\
37.25 \\
116.64\end{array}$ & $\begin{array}{r}1.00 \\
5.97 \\
30.76 \\
94.61 \\
31.34 \\
95.66\end{array}$ & $\begin{array}{r}0.16 \\
2.18 \\
25.13 \\
37.57 \\
64.75 \\
67.69\end{array}$ & $\begin{array}{rr}0.19 \\
3 \\
2.49 \\
28.81 \\
43.33 \\
76.41 \\
78.66\end{array}$ \\
\hline $\begin{array}{l}\text { Total das Doenças } \\
\text { Cardiovasculares }\end{array}$ & 199.37 & 319.18 & 344.55 & 294.37 & 315.56 & 259.34 & 197.44 & + 229.89 \\
\hline Todas as Causas & 772.44 & 1007.37 & 1086.40 & 997.33 & 936.27 & 853.16 & 715.42 & 787.92 \\
\hline $\begin{array}{l}\text { Proporção de óbitos por } \\
\text { Sint. e Estado Morb. Mal. Def. (3) }\end{array}$ & & .00 & 1.8 & & 2.3 & 39 & & 9.94 \\
\hline
\end{tabular}

(1) Padronização por idade - Método indireto - estrutura de taxas padrão:estado do Rio Grande do Sul (1970-1975).

(2) Inclui todos os municípios do estado do Rio de Janeiro, c/exceção do município do Rio de Janeiro.

(3) Proporção em percentual (\%) dos óbitos por sintomas e estados mórbidos mal definidos em relação a todos os óbitos.

Portanto, em função das características de industrialização e da importância das doenças cardiovasculares em Volta Redonda, pareceu ser relevante um estudo sobre hiperten- 
são arterial na sua população. A escolha da hipertensão arterial está relacionada com o fato de ser um dos fatores de risco mais importantes para o desenvolvimento de doenças isquêmicas do coração e cerebrovasculares ${ }^{17},{ }^{18}$, as duas maiores causas de óbito no país ${ }^{12}$, e, com a experiência adquirida a respeito pelos autores em estudo anterior, no estado do Rio Grande do Sul, Brasil 2, 3, 4, 5, 11,15. Deste modo, foi feito um inquérito epidemiológico, um estudo seccional de população, em Volta Redonda, com a finalidade de estimar a prevalência de hipertensão arterial nos adultos e examinar possíveis associações entre alguns fatores e a doença. A coleta de informações no campo foi realizada no período de novembro de 1979 a janeiro de 1980 .

Esta pesquisa teve seu suporte financeiro e técnico fornecido inicialmente pelo Hospital da Sidenúrgica Nacional de Volta Redonda, na fase de análise pela Secretaria de Ciência e Tecnologia do Ministério da Saúde, e, ao longo de todo período, pela Escola Nacional de Saúde Pública (Fundação Oswaldo Cruz).

\section{TABELA II}

Mortalidade proporcional, por 100 óbitos, por causas cardiovasculares, por sintomas e estados mórbidos mal definidos e por todas as causas, no estado do Rio de Janeiro, no periodo de 1968-1972.

\begin{tabular}{lcrrr}
\hline \multicolumn{1}{c}{ Municípios } & $\begin{array}{c}\text { Volta } \\
\text { Redonda }\end{array}$ & Niterói & $\begin{array}{c}\text { Município do } \\
\text { Rio de Janeiro }\end{array}$ & $\begin{array}{c}\text { Estado do } \\
\text { Rio (1) }\end{array}$ \\
Causas de Óbito & 0.04 & 0.07 & 0.10 & 0.02 \\
\hline Febre Reumática Ativa & 0.52 & 0.73 & 0.72 & 0.30 \\
Doença Reum. Crôn. do Cor. & 4.40 & 3.59 & 4.05 & 3.51 \\
Doenças Hipertensivas & 5.00 & 9.21 & 12.40 & 5.25 \\
Doenças Isquêmicas Coração & 5.97 & 8.24 & 3.98 & 9.46 \\
Outras Formas Doença Cor. & 9.87 & 10.44 & 12.46 & 27.60 \\
Doença Cerebrovascular & & & & \\
Todas as Causas & 25.80 & 31.71 & 33.70 & 19.94 \\
Cardiovasculares & & & & 2.39 \\
Sintomas e Estados & 17.00 & 1.86 & 100.00 & 100.00 \\
Mórbidos Mal Definidos & 100.00 & 100.00 & & \\
Todas as Causas & & & & \\
\hline
\end{tabular}

(1) Inclui todos os municípios do estado do Rio de Janeiro, com exceção do município do Rio de Janeiro.

\section{METODOLOGIA}

Foi realizado um estudo epidemiológico seccional (ou inquérito populacional), cuja população alvo era aquela reśidente na cidade de Volta Redonda. A coleta de informações foi feita por quatro auxiliares de enfermagem, supervisionada por um médico, no período de novembro de 1979 a ja- 
neiro de 1980, em domicílios sorteados aleatoriamente, onde eram entrevistados todos os adultos de 20 a 74 anos de idade.

\section{A AMOSTRA}

Não existiam listagens que compreendessem o universo dos residentes em Volta Redonda. A listagem mais abrangente era a dos prédios da área urbana do cadastro do Serviço Autônomo de Água e Esgoto da Prefeitura Municipal. Segundo estatísticas recentes do próprio serviço, em 1977, cerca de $90 \%$ dos domicílios eram servidos pela rede pública de água. Assim, foi decidido utilizar-se essa listagem de domicílios para, através dela, obter-se uma amostra representativa da cidade de Volta Redonda, por meio da técnica de amostragem aleatória simples. Portanto, a amostra obtida deveria representar apenas a área urbana (98\% do município).

O tamanho da amostra necessário, em indivíduos, foi estimado para uma prevalência suposta de hipertensão arterial de $20 \%(p=0.2)$. Assim, para um intervalo de confiança de $95 \%\left(t_{\alpha}=1.96\right)$ um erro fixado de $3 \%(d)$, e um efeito de delineamento de $40 \%(E=1.4)$, o tamanho da amostra seria de 956 pessoas $\left(n=\left(t_{\alpha}{ }^{2} \cdot p \cdot(1-p) \cdot E\right) / d^{2}\right)$. Esse efeito de delineamento se deve à suposição de que a variância total pode ter sido reduzida em até $40 \%$ (o que certamente é uma estimativa conservadora), uma vez que os indivíduos foram selecionados em aglomerados, os domicílios, nos quais há maior homogeneidade do que na população. Para efeitos práticos, o tamanho de amostra necessário, em termos de indivíduos, foi igualado a 1000 . O censo de $1970^{7}$ estimava que cada domicílio particular era habitado por cerca de 2.5 adultos, portanto 1000 indivíduos seriam encontrados em 400 domicílios. Considerando que, provavelmente, cerca de $90 \%$ dos domicílios deveriam ser efetivamente habitados, seriam necessários $\mathbf{4 4 4}$ domicílios. Porém, como a listagem enumerava todos os prédios, e não somente os domicílios particulares, foram sorteados 555 endereços, já que, segundo o próprio serviço, cerca de $80 \%$ dos endereços corresponderiam a domicílios.

As listagens foram ordenadas segundo bairros e ruas, e os endereços enumerados em ordem crescente ( 1 a 37034). Com o auxílio de uma tábua de números aleatórios ${ }^{6}$ foram sorteados os endereços.

\section{Os Questionários e as Medidas}

Em cada domicílio sorteado deveriam ser entrevistados, por uma auxiliar de enfermagem, todos os adultos de 20 a 74 anos de idade.

Foram utilizados três questionários. $\mathrm{O}$ primeiro, a Ficha Domiciliar, éra único para cada domicílio. Esta ficha con- 
tém informações sobre os resultados de cada visita, as características gerais do domicílio, a classificação an tropogenética da família e uma relação completa dos moradores. $\mathrm{O}$ segundo, a Ficha Individual, contém informações sobre migração, dados biológicos e demográficos básicos, situação social e ocupacional, hábitos (fumo, bebida alcoólica e esporte) e itens médicos (história familiar e revisão diagnóstica), prestadas por cada um dos adultos participantes do estudo. $\mathrm{O}$ terceiro, a Ficha de Medidas, contém as informações individuais sobre pressão arterial, pulso periférico, circunferência braquial, temperatura ambiente e dosagens de sódio e creatina em amostras casuais de urina (o peso e a altura não puderam ser registrados por dificuldades no transporte de equipamentos).

A medida de pressão arterial de cada indivíduo foi feita duas vezes em uma mesma ocasião. A primeira medida era feita imediatamente após o primeiro contato com cada pessoa, e, a segunda, cerca de 30 minutos depois, sendo que no intervalo entre as duas medidas os indivíduos deveriam permanecer em repouso relativo, isto é, sentados, sem fumar, nem beber café ou outro estimulante, apenas respondendo às questões da Ficha Individual. As medidas foram feitas no braço esquerdo, com o mesmo apoiado sobre uma mesa, e o indivíduo sentado, tendo sido registrados os pontos correspondentes à sistólica (fase I) e à primeira e à segunda diastólicas (fases IV e V, respectivamente). Os equipamentos utilizados foram quatro esfigmomanômetros de coluna de mercúrio, portáteis, da marca Tycos (HRI 8104. 509701), porém modificados (ENPS-II) ${ }^{4}$. Estes aparelhos já haviam sido testados pelo uso em pesquisa anterior, e suas modificações são baseadas na desconexão audiovisual e na estabilização da velocidade de deflação, que resultam em atenuação de variações e erros próprios do observador. $\mathrm{O}$ painel que contém a escala de leitura do aparelho original foi substituído por outro, que só permite a leitura pelo acionamento de um dispositivo de iluminação, colocado atrás do painel, com uma das mãos, o que torna pratica. mente impossível o registro de um valor durante a queda da coluna de mercúrio. Portanto, para se poder fazer uma leitura, é preciso obstruir completamente a saída de ar do manguito, de uma maneira simples e rápida. Isto foi obtido através da modificação da válvula que só tem duas posições: aberta, mantida por pressão do polegar, e, fechada, por inércia. Esta característica não permite variações da velocidade de deflação do manguito impostas pelo observador. A velocidade média de deflação entre 200 e $30 \mathrm{mmHg}$ era de $2 \mathrm{~mm} /$ segundo, sendo que, na faixa de 200 a $100 \mathrm{mmHg}$, era de $2.7 \mathrm{e}$, na de 100 a 30, era de $1.3 \mathrm{~mm} /$ segundo. Essas modificações visam fazer com que o examinador, durante a medida, se ocupe apenas com a identificação auditiva dos sons de Korotkow, sendo que, ao identificar cada um dos 
pontos convencionados, início, abafamento e cessação de batimentos, lhe seja possível afastar a mão da válvula, acionar o dispositivo de iluminação da escala, fazer a leitura e anotar o valor observado. As medidas foram feitas com intervalos de $2 \mathrm{mmHg}$, com arredondamentos para o valor mais próximo.

0 pulso periférico radial foi medido com o auxílio de um cronômetro (Hanhart West Germany), após cada medida de pressão arterial, sendo registrado o tempo decorrido entre 30 batimentos.

A circunferência braquial foi medida com fita métrica de nylon no ponto médio do braço, entre o acrômio e o olécrano, em milímetros.

As dosagens de sódio e creatina foram feitas em amostras de urina, coletadas entre as duas medidas de pressão, em frascos de $50 \mathrm{~cm}^{3}$, contendo ácido bórico, com espectrofotômetro, no Hospital da Siderúrgica Nacional.

A temperatura ambiente foi medida em centígrados com termômetro de coluna de álcool, colocado no local das medidas.

\section{Os Observadores}

As medidas e entrevistas foram feitas por quatro auxiliares de enfermagem, selecionadas de um grupo maior, e treinadas durante duas semanas para se obter a melhor padronização.

Uma atenção especial foi dada ao treinamento da medida de pressão arterial. É sabido que os observadores podem distorcer ou aumentar a variabilidade da pressão arterial através de: a) preferência inconsciente por pressões com determinado dígito terminal (habitualmente zero); b) preconceitos formados por avaliação prévia do examinado; c) aproximações para medidas tidas como mais freqüentes; e, d) variações na velocidade de desinflação do manguito ${ }^{10}, 20,21$ As modificações do esfigmomanômetro utilizado pretendem corrigir estes erros. $O$ treinamento da medida de pressão arterial foi feito através de instrução individual, e, com o auxílio de uma fita magnética gravada com séries de sons de Korotkow, fornecida pelo professor Geoffrey Rose (London School of Hygiene and Tropical Medicine). A fita, tocada num gravador $\mathrm{K}-7$ comum, reproduz situações reais de audição dos sons de Korotkow, representando o indivíduo observado, e três cronômetros são utilizados para representar o instrumento de medida.

As demais medidas foram treinadas através de séries contínuas de simulação para avaliação de precisão e exatidão, até a observação do máximo de padronização entre observadores, pelo método sugerido por Habicht ${ }^{9}$.

Cada domicílio era visitado por um dos entrevistadores, que seguia uma conduta padronizada por um roteiro escrito. A distribuição de endereços para cada entrevistador era 
feita por um supervisor em grupos correspondentes a bairros. $O$ supervisor sorteava, ainda, semanalmente, 1/10 dos domicílios visitados para proceder a uma revisão das informações tomadas pelas auxiliares, no local. Algumas informações selecionadas foram transferidas para uma ficha resumo, que foi utilizada para análises rápidas ainda durante a fase de coleta, a fim de controlar a qualidade dos dados e, também, para informes preliminares publicados ${ }^{13}, 14$.

Finalmente, após o encerramento da fase de campo, todos os dados foram transferidos para fitas magnéticas, através de computador. Esses dados foram organizados em um arquivo, segundo os indivíduos, e tiveram sua consistência testada por programas de verificação. Este trabalho foi feito no Serviço de Processamento de Dados da Fundação Oswaldo Cruz e, posteriormente, no Núcleo de Computação da Universidade Federal do Rio de Janeiro, onde também são processados os dados para análises estatísticas.

\section{RESULTADOS E COMENTÁRIOS}

\section{Cobertura da Amostra}

Dos 555 endereços planejados, 444 correspondiam a domicílios particulares e, entre estes, 429 eram habitados, constituindo-se, portanto, na amostra efetiva. Destes domicílios, $8.2 \%$ foram perdidos por recusa de participação, $7.2 \%$ por estarem fechados em três visitas e $13.3 \%$ por outras razões. No final, foram cobertos 305 domicílios habitados, com 650 adultos.

Repare-se que, daqui por diante, as tabelas de resultados, em geral, apresentam totais de indivíduos menores do que 650. Isto se deve, ainda, a perdas parciais de algumas informações, para alguns indivíduos. Assim, por exemplo, não foi possível obter a segunda medida de pressão arterial de quatro pessoas, que saíram de casa durante a entrevista.

\section{A Prevalência da Hipertensão Arterial}

O primeiro objetivo do estudo era estimar a prevalência da hipertensão arterial na área urbana de Volta Redonda. 0 critério utilizado para o diagnóstico de hipertensão arterial foi da $\mathrm{OMS}^{23}$, que classifica como hipertensos os indivíduos cuja pressão sistólica seja igual ou maior do que 160 e/ou cuja pressão diastólica seja igual ou maior do que $95 \mathrm{mmHg}$. Neste estudo utilizou-se para diagnóstico apenas os valores obtidos na segunda medida de pressão arterial, aquela em repouso relativo, e, para a diastólica, apenas os valores da segunda diastólica (fase $\mathbf{V}$ ou de desaparecimento dos sons de Korotkow).

A prevalência da HA nos adultos de 20 a 74 anos de idade, da área urbana de Volta Redonda, foi estimada em $10.1 \%$ na amostra examinada. A variância dessa proporção 
foi estimada numa amostra de aglomerados (domicílios), portanto é preciso levar em consideração o efeito de delineamento na estimativa da variância ${ }^{1}$. $O$ coeficiente de correlação intraclasse (intradomicílio) estimado, através de análise de variância, foi de 0.208 , portanto, a variância corrigida será dada por $S_{c}^{2}=S^{2}$. E, em que E é o efeito de delineamento, obtido por $\mathrm{E}=1+\mathrm{r}(\overline{\mathrm{n}}-1)$, onde $\mathrm{r}$ é o coeficiente de correlação intraclasse e $\bar{n}$ é o número médio de observações em cada classe (média de indivíduos por domicílio). $O$ valor de $E$ na amostra foi de $1.2^{\wedge} 3$, e, deste modo, o intervalo de confiança de $95 \%$ para a prevalência de HA está contido entre os limites de 7.5 e $12.7 \%$ (considerando-se que as perdas de observação não estão relacionadas com a pressão arterial).

TABELA III - 1

Prevalência de hipertensão arterial (\%) segundo sexo e grupo etário - Volta Redonda, 1979-1980.

\begin{tabular}{lrrrrrr}
\hline \multicolumn{1}{r}{ Sexo } & \multicolumn{2}{c}{ Homens } & \multicolumn{2}{c}{ Mulheres } & \multicolumn{2}{c}{ Todos } \\
\hline Grupo Etário & $\mathrm{p}(\%)$ & $\mathrm{n}$ & $\mathrm{p}(\%)$ & $\mathrm{n}$ & $\mathrm{p}(\%)$ & $\mathrm{n}$ \\
\hline $20-29$ & 1.0 & 100 & 0.7 & 137 & 0.8 & 237 \\
$30-39$ & 9.2 & 65 & 8.8 & 68 & 9.0 & 133 \\
$40-49$ & 20.0 & 45 & 18.4 & 76 & 19.0 & 121 \\
$50-59$ & 16.7 & 48 & 11.9 & 42 & 14.4 & 90 \\
$60-74$ & 17.2 & 29 & 27.8 & 36 & 23.1 & 65 \\
Todos & 10.1 & 287 & 10.0 & 359 & 10.1 & 646 \\
\hline
\end{tabular}

A tabela III-1 apresenta prevalência de HA em grupos, segundo sexo e idade. Estes dados sugerem que a prevalência de HA cresce de forma acentuada dos 20 aos 49 anos, porém que a partir daí se estabiliza ou, pelo menos, tem seu crescimento atenuado. Entretanto, é preciso ter cuidado ao interpretar dados de um estudo seccional, pois cada coorte de idade é examinada apenas em um momento do tempo. Assim, os jovens não necessariamente representam a juventude dos velhos ou, de outro modo, os velhos não precisam representar a velhice dos jovens no momento do estudo. De qualquer modo, a tendência revelada em Volta Redonda não é comum com os resultados de outros estudos da mesma natureza $\mathrm{a}^{4},{ }^{16}$, pois, em geral, a prevalência de HA cresce de modo contínuo, em ambos os sexos. Em comparação com os resultados de uma pesquisa, com metodologia muito semelhante, feita no estado do Rio Grande do Sul, em 1978, as prevalências em Volta Redonda são mais elevadas até os 49 anos, porém são semelhantes ou mais baixas daí em diante, tanto nas mulheres como nos homens, porém mais especialmente nestes últimos. Se diferenças devidas a erros de classificação e a flutuações amostrais (são pequenos os denominadores utilizados para as estimativas específicas, por sexo e idade) pudessem ser descartadas, pode- 
ria se pensar na existência de maiores riscos mais precoces em Volta Redonda, onde os mais jovens têm prevalências mais elevadas. Porém, devido a fatores de seleção, para sobrevivência até idades mais avançadas, mais atuantes em Volta Redonda, os mais velhos passariam a ter prevalências mais baixas. Essa hipótese poderia ser reforçada pela comparação entre as taxas de mortalidade, segundo sexo e idade, especialmente aquelas devidas a causas de óbito do aparelho cardiovascular. Infelizmente não dispomos dos óbitos, por sexo, idade, e por grupo de causas em Volta Redonda, porém a sua taxa de mortalidade global por causas cardiovasculares é maior do que a do Rio Grande do Sul, quando ajustada pela estrutura etária (método indireto). Assim, a taxa de mortalidade por causas cardiovasculares era de $319.2^{\circ} / 0000$ em Volta Redonda (1968-72), e de 193.8\%/0000 no estado do Rio Grande do Sul $(1969 / 75)^{22}$. Por fim, dados disponíveis para o ano de 1970 indicam que a mortalidade geral no grupo etário de 20 a 49 anos é maior em Volta Redonda do que no Rio Grande do Sul (4.58 e $2.71^{\circ} / 00$, respectivamente), porém, no grupo etário de 50 anos e mais a diferença relativa é atenuada (32.16 e $27.82^{0} / 00$, respectivamente) ${ }^{19,22}$. Portanto, podemos supor que a seleção para sobrevivência atuou mais precocemente em Volta Redonda, retirando mais cedo da população aqueles indivíduos afetados pela hipertensão arterial, que, afinal, representa um risco mais elevado de óbito por causas cardiovasculares.

\section{Médias das Pressões Sistólicas e Diastólicas}

A média estimada da pressão Sistólica foi de 122.4 $\mathrm{mmHg}$, nos adultos de 20 a 74 anos de idade, da área urbana de Volta Redonda. $\mathrm{O}$ efeito de delineamento, por aglomeração domiciliar, foi estimado na amostra em 1.184 (o coeficiente de correlação intraclasse foi de 0,165 ). Portanto, a variância da média corrigida foi de 0.753 , e os limites do intervalo de confiança de $95 \%$ estão contidos entre 120.7 e $124.1 \mathrm{mmHg}$.

A média estimada da pressão diastólica-2 (face $V$ ) foi de $70.6 \mathrm{mmHg}$, nos adultos de 20 a 74 anos de idade, da área urbana de Volta Redonda. O efeito de delineamento, por aglomeração domiciliar, foi estimado na amostra em 1.411 (o coeficiente de correlação intraclasse foi de 0.368 ). Portanto, a variância da média, corrigida, foi de 0,548 , e os limites do intervalo de confiança de $95 \%$ estão contidos entre 69.1 e $72.1 \mathrm{mmHg}$.

As tabelas III-2 e III-3 apresentam as médias das pressões segundo sexo e idade. A sistólica tem relações semelhantes com a idade àquelas existentes entre a prevalência e a idade de ambos os sexos. Já a diastólica apresenta relação positiva com a idade até cerca de 49 anos, sendo que, a partir daí, 
sua tendência é a estabilização e queda. Novamente, nas comparações com a amostra estudada no Rio Grande do Sul, as tendências são as mesmas descritas para a prevalência, com exceção da sistólica nas mulheres, que apresentam médias mais baixas em Volta Redonda, em praticamente todas as faixas etárias.

No artigo que se segue iremos analisar as relações entre fatores selecionados e a pressão arterial.

\section{TABELA III-2}

Médias de pressão sistólica, segundo sexo $e$ grupo etário - Volta Redonda, 1979-1980.

\begin{tabular}{l|rrr|rrrrrrrrr}
\hline \multicolumn{1}{r}{ Sexo } & \multicolumn{3}{c}{ Homens } & \multicolumn{3}{c}{ Mulheres } & \multicolumn{3}{c}{ Todos } \\
\hline Grupo & - & & & & & & & & & & & \\
Etário & $\mathrm{x}$ & $\mathrm{s}$ & $\mathrm{n}$ & $\mathrm{x}$ & $\mathrm{s}$ & $\mathrm{n}$ & $\mathrm{x}$ & $\mathrm{s}$ & $\mathrm{n}$ \\
\hline $20-29$ & 117.0 & 12.9 & 100 & 108.9 & 12.3 & 137 & 112.3 & 13.2 & 237 \\
$30-39$ & 125.0 & 14.4 & 65 & 118.7 & 20.8 & 68 & 121.8 & 18.1 & 133 \\
$40-49$ & 126.8 & 19.1 & 45 & 127.3 & 23.0 & 76 & 127.1 & 21.6 & 121 \\
$50-59$ & 134.1 & 21.3 & 48 & 128.5 & 19.6 & 42 & 131.5 & 20.6 & 90 \\
$60-74$ & 135.2 & 22.9 & 29 & 142.7 & 22.8 & 36 & 139.4 & 23.0 & 65 \\
Todos & 125.1 & 18.3 & 287 & 120.3 & 21.5 & 359 & 122.4 & 20.3 & 646 \\
\hline
\end{tabular}

TABELA III -3

Médias de pressão diastólica, segundo sexo $e$ grupo etário - Volta Redonda, 1979-1980.

\begin{tabular}{l|rrrrrrr|rrrrr}
\hline \multicolumn{1}{r}{ Sexo } & \multicolumn{3}{r}{ Homens } & \multicolumn{3}{c}{ Mulheres } & \multicolumn{3}{c}{ Todos } \\
\hline Grupo & - & & & - & & & & & \\
Etário & $\bar{x}$ & $\mathrm{~s}$ & $\mathrm{n}$ & $\mathrm{x}$ & $\mathrm{s}$ & $\mathrm{n}$ & $\mathrm{x}$ & $\mathrm{s}$ & $\mathrm{n}$ \\
\hline $20-29$ & 67.4 & 12.2 & 100 & 62.7 & 12.1 & 137 & 64.6 & 12.3 & 237 \\
$30-39$ & 72.8 & 14.4 & 65 & 71.0 & 17.0 & 68 & 71.9 & 15.7 & 133 \\
$40-49$ & 76.3 & 17.7 & 45 & 75.0 & 19.2 & 76 & 75.5 & 18.6 & 121 \\
$50-59$ & 77.1 & 15.3 & 48 & 73.2 & 14.8 & 42 & 75.3 & 15.1 & 90 \\
$60-74$ & 73.2 & 17.1 & 29 & 74.7 & 15.9 & 36 & 74.0 & 16.3 & 65 \\
Todos & 72.2 & 15.1 & 287 & 69.3 & 16.3 & 359 & 70.6 & 15.8 & 646 \\
\hline
\end{tabular}

\section{CONCLUSÕES}

A hipertensão arterial é um problema de massas em Volta Redonda, pois cerca de 1 em cada 10 adultos é afetado pelo mal, que é um dos riscos mais importantes para o desenvolvimento de afecções cardiovasculares tais como as doenças isquêmicas e os acidentes cerebrovasculares.

Em Volta Redonda são elevadas as prevalências de hipertensão arterial nos adultos jovens até a meia-idade, quando comparadas com aquelas obtidas em outros locais. Isto sugere que, em Volta Redonda, os fatores de risco para HA atuam precocemente durante a vida das pessoas. 
No final do ano de 1979 foi realizado um estudo seccional, numa amostra probabilistica de domicilios, da cidade de Volta Redonda, estado do Rio de Janeiro, sobre Hipertensão Arterial. Foram examinados 650 adultos, de 20 a 74 anos de idade, nos seus domicilios. $O$ artigo descreve a metodologia empregada para a tomada de informações e aponta alguns resultados gerais.

As médias das pressões sistólica e diastólica foram de $122.4 \pm 0.9$ e $70.6 \pm 0.7 \mathrm{mmHg}$, respectivamente, enquanto que a prevalência de Hipertensão Arterial, segundo o critério da OMS /sistólica $\geqslant 160$ e/ou diastólica $\geqslant$ $95 \mathrm{mmHg}$ ), foi de $10.1 \pm 1.3 \%$ considerado o efeito de delineamento amostral por aglomeração domiciliar.

Em Volta Redonda, as prevalências de Hipertensão Arterial são elevadas nos adultos jovens e de meia-idade, especialmente nos homens, o que se traduz em altas taxas de mortalidade por doenças do sistema cardiocirculatório nestes grupos etários.

\section{REFERÊNCIAS BIBLIOGRĀFICAS}

1. BLALOCK, H.M. Social statistics. Tokyo, McGrawHill, 1979.

2. COSTA, E.A. et alii. A cross-sectional survey of blood pressure in Rio Grande do Sul - Brazil, 1978. In: SCIENTIFIC MEETING OF THE INTERNATIONAL EPIDEMIOLOGICAL ASSOCIATION, 9. Edinburgh, Scotland, 1981.

3. COSTA, E.A. et alii. Hipertensão arterial no estado do Rio Grande do Sul. Ci. Cult., 32 (supl.) :67, jul. 1980. [Apresentado na 32ạ Reunião Anual da SBPC, Rio de Janeiro, 1980].

4. COSTA, E.A. et alii. Pesquisa epidemiológica sobre hipertensão arterial no Rio Grande do Sul; ou Hipertensão arterial uma perspectiva sócio-ecológica. Rio de Janeiro, PEPPE/FIOCRUZ, 1980.

5. COSTA, E.A. et alii. Salt and blood pressure in Rio Grande do Sul - Brazil, 1978. In: SCIENTIFIC MEETING OF THE INTERNATIONAL EPIDEMIOLOGICAL ASSOCIATION, 9. Edinburgh, Scotland, 1981.

6. FLEISS, J.L. Statistical methods for rates and proportions. New York, John Wiley \& Sons, 1973.

7. FUNDAÇÃO INSTITUTO BRASILEIRO DE GEOGRAFIA E ESTATÍSTICA. Censo demográfico; Rio de Janeiro; 89 recenseamento geral do Brasil 
- 1970. Rio de Janeiro, IBGE, 1973. (Série regional, v. 1, t. 16).

8. FUNDAÇÃO INSTITUTO BRASILEIRO DE GEOGRAFIA E ESTATÍSTICA. Sinopse preliminar do censo demográfico; Rio de Janeiro; $99^{9}$ recenseamento geral - 1980. Rio de Janeiro, IBGE, 1981. (v. 1, t. 1, n. 17).

9. HABICHT, J.P. Estandarización de métodos epidemiológicos cuantitativos sobre el terreno. Bol. Of. Sanit. Panam., 76 (5) :375-84, 1974.

10. HOLLAND, W.W. The reduction of observer variability in the measurement of blood pressure. In: PEMBERTON, J. (ed.) Epidemiology; reports on research and teaching. London, Oxford University Press, 1962.

11. KLEIN, C.H. Hipertensão arterial em estratos geoeconômicos do Rio Grande do Sul. Rio de Janeiro, 1981. (Tese de Mestrado - ENSP).

12. KLEIN, C.H. Panorama das doenças cardiovasculares no Brasil. Arq. bras. Cardiol., 32 (supl. 2) :181, 1979.

13. KLEIN, C.H. et alii. Hipertensão arterial em Volta Redonda. Ci. Cult., 32 (supl.) :67, jul. 1980. [Apresentado na 32a Reunião Anual da SBPC, Rio de Janeiro, 1980].

14. KLEIN, C.H. et alii. Hipertensão arterial em Volta Redonda. An. Hosp. Sider. Nac., 4 (2) :59-64, 1980.

15. KLEIN, C.H. et alii. Socio-economic variables and blood pressure in Rio Grande do Sul. In: SCIENTIFIC MEETING OF THE INTERNATIONAL EPIDEMIOLOGICAL ASSOCIATION, 9. Edinburgh, Scotland, 1981.

16. PAUL, O. Epidemiology of hypertension. In: GROSS, F.H. \& ROBERTSON, J.S. (ed.) Arterial hypertension. London, Pitman Med. Publ., 1979.

17. PICKERING, G. Hypertension; definitions, natural histories and consequences. Amer. J. Med., (52) :570-83, 1972.

18. PUFFER, R.R. \& GRIFFITH, G.W. Patterns of urban mortality. Washington, PAHO, 1968. (Scientific Publication, 151).

19. RIO DE JANEIRO (estado). Secretaria de Planejamento e Coordenação Geral \& FUNDAÇÃO INSTITU. TO DE DESENVOLVIMENTO ECONOMICO E SOCIAL. Estado do Rio de Janeiro; estatísticas de sistemas de saúde. Rio de Janeiro, FIDERJ/DIGES/ SIPE, 1977. 
20. ROSE, G. Standardization of observers in blood pressure measurement. Lancet, $1: 673-5,1975$.

21. ROSE, G. et alii. A sphygnomanometer for epidemiologists. Lancet, $1: 296-7,1964$.

22. RIO GRANDE DO SUL. Secretaria de Saúde. Estatisticas de sauide; mortalidade 1970-75. Porto Alegre, 1977. v. 1.

23. WORLD HEALTH ORGANIZATION. Arterial hypertension. Geneva, 1978. (Technical report series, 628). 